\title{
A new angle on dynamic depolarized light scattering: number-averaged size distribution of nanoparticles in focus $\uparrow$
}

\author{
Christoph Geers, ${ }^{a}$ Laura Rodriguez-Lorenzo, ${ }^{a}$ Dominic Andreas Urban, ${ }^{a}$ \\ Calum Kinnear, ${ }^{a, b, c}$ Alke Petri-Fink ${ }^{a, b}$ and Sandor Balog ${ }^{\star a}$
}

\begin{abstract}
Size polydispersity is a common phenomenon that strongly influences the physicochemical properties of nanoparticles (NPs). We present an analytical approach that is universally applicable to characterizing optically anisotropic round NPs and determines directly the number-averaged size distribution and polydispersity via depolarized dynamic light scattering (DDLS). To demonstrate, we use aqueous suspensions of Au NPs of different sizes and surface functionalization.
\end{abstract}

\section{Introduction}

The physicochemical properties of nanoparticles (NPs), which are present in several fields of research and applications, ${ }^{1}$ are size-dependent. These include catalytic, ${ }^{2,3}$ magnetic $^{4}$ and optical properties, ${ }^{5}$ visual appearance and colour, ${ }^{6}$ self-assembly, ${ }^{7,8}$ aggregation and clustering, ${ }^{9,10}$ behaviour at the interface between nanomaterials and biological systems ${ }^{11}$ as well as the adsorption of molecules and proteins, ${ }^{12,13}$ in vitro cellular dose ${ }^{14,15}$ cellular endocytosis ${ }^{16}$ and toxicity. ${ }^{17}$ Particle polydispersity is a common phenomenon that influences the sizedependent physicochemical properties. Given that hundreds of papers report on the successful control over NP size, shape, composition, and surface chemistry, the concern regarding accuracy and reproducibility has come as no surprise. The need for advanced characterization has become critical, ${ }^{18}$ and establishing accurate experimental protocols dedicated to the in situ characterization of the number-averaged size distribution of such NPs suspended in e.g. aqueous biological and physiological environments is of considerable interest. ${ }^{19,20}$ Dynamic depolarized light scattering (DDLS) potentially offers clear advantages over competing techniques, especially when it comes to probing NPs in situ in complex biological and physiological fluids. ${ }^{21}$ An inherent but often critical feature of scatter-

${ }^{a}$ Adolphe Merkle Institute, University of Fribourg, Chemin des Verdiers 4, 1700 Fribourg, Switzerland. E-mail: sandor.balog@unifr.ch

${ }^{b}$ Chemistry Department, University of Fribourg, Chemin du Musée 9, 1700 Fribourg, Switzerland

${ }^{c}$ Institute \& School of Chemistry, The University of Melbourne, 30 Flemington Rd3010, Australia

$\dagger$ Electronic supplementary information (ESI) available. See DOI: 10.1039/ c6nr03386e ing experiments using biological and physiological fluids is that the measured primary signal, that is, the scattering intensity, contains contributions from proteins. These contributions to the scattering intensity may be significant; however, separating the relevant signals from the irrelevant ones is not trivial, which hinders the effective usage of DLS for investigating NPs in these application-relevant environments. Relying on depolarization, unwanted scattering signals from proteins that are not associated with the NPs can be successfully suppressed with an unprecedented signal-to-noise ratio. DDLS relies on optical anisotropy, which may originate from shape or internal anisotropy. Any particle - whether dielectric or metallic - possessing either one of those, is suitable for DDLS. Yet, little is known about the quantitative interpretation of DDLS spectra with regard to number-averaged size distribution, other than the fact that the field correlation function from polydisperse samples may be expressed as the Laplace transform of the probability density function describing the dispersion in the relaxation rate: $:^{22,23}$

$$
\begin{aligned}
g_{1}(t) & =\int_{0}^{\infty} P(\Gamma) \mathrm{e}^{-\Gamma t} \mathrm{~d} \Gamma=\sum_{j=0}^{\infty} \frac{1}{j !}\left\langle\Gamma^{j}\right\rangle t^{j} \\
& =1-\langle\Gamma\rangle t+\frac{1}{2}\left\langle\Gamma^{2}\right\rangle t^{2}-\frac{1}{6}\left\langle\Gamma^{3}\right\rangle t^{3}+\ldots
\end{aligned}
$$

where $P(\Gamma)$ is the probability density function of the relaxation rate, which is the function of the particle radius: $\Gamma(q, r)=6 D_{\mathrm{R}}+$ $q^{2} D_{\mathrm{T}}$, where $D_{\mathrm{R}}=\frac{k_{\mathrm{B}} T}{8 \pi \eta} \frac{1}{r^{3}}$ and $D_{\mathrm{T}}=\frac{k_{\mathrm{B}} T}{6 \pi \eta} \frac{1}{r}$ correspond to translational $\left(D_{\mathrm{T}}\right)$ and rotational $\left(D_{\mathrm{R}}\right)$ diffusivity, respectively. ${ }^{24-28}$ $\left\langle\Gamma^{j}\right\rangle \equiv \int_{0}^{\infty} P(\Gamma) \Gamma^{j} \mathrm{~d} \Gamma$ is the $j^{\text {th }}$ raw moment of $P(\Gamma)$, and $j$ is a nonnegative integer, $k_{\mathrm{B}}$ is the Boltzmann constant, $T$ is the temperature, $\eta$ is the viscosity of the solvent, $q$ is the momen- 
tum transfer $q=\frac{4 \pi}{\lambda} n \sin \left(\frac{\theta}{2}\right), \theta$ is the scattering angle, $\lambda$ is the wavelength of the scattered waves, and $n$ is the refractive index of the suspension. While eqn (1) is a straightforward representation, its practicality falls short because $P(\Gamma)$ and $\left\langle\Gamma^{j}\right\rangle$ represent only the distribution and raw moments weighted by the scattering intensity. These are generally not relevant when considering the above-mentioned size-dependent physicochemical properties of NPs, and if possible they are to be converted to number-averaged values..$^{29-31}$ We introduce here an approach for DDLS: by expressing $\left\langle\Gamma^{j}\right\rangle$ as a function of the raw moments of the particle size distribution, the correlation function can be expressed via another polynomial, whose coefficients are now functions of the raw moments of the number-averaged particle size distribution (ESI, $\uparrow$ Correlation function of depolarized scattering from polydisperse nanoparticles). Accordingly,

$$
\begin{aligned}
g_{1}(t)=1 & -\frac{k_{\mathrm{B}} T}{\pi \eta}\left(\frac{6}{8} \frac{\left\langle r^{3}\right\rangle}{\left\langle r^{6}\right\rangle}+\frac{q^{2}}{6} \frac{\left\langle r^{5}\right\rangle}{\left\langle r^{6}\right\rangle}\right) t \\
& +\left(\frac{k_{\mathrm{B}} T}{\pi \eta}\right)^{2}\left(\frac{9}{32} \frac{1}{\left\langle r^{6}\right\rangle}+\frac{q^{2}}{8} \frac{\left\langle r^{2}\right\rangle}{\left\langle r^{6}\right\rangle}+\frac{q^{4}}{72} \frac{\left\langle r^{4}\right\rangle}{\left\langle r^{6}\right\rangle}\right) t^{2} \\
& +\ldots
\end{aligned}
$$

where $\left\langle r^{j}\right\rangle \equiv \int_{0}^{\infty} P(r) r^{j} \mathrm{~d} r$ is the $j^{\text {th }}$ raw moment, and $P(r)$ is the probability density distribution of the particle radius. It is important to mention that eqn (1) is not specific only to DDLS, and as long as the particles exhibit moderate polydispersity, the ratios of raw moments in eqn (2) may be substituted for the corresponding average size, that is, $\left\langle r^{6}\right\rangle /\left\langle r^{3}\right\rangle \cong\langle r\rangle^{3}$ and $\left\langle r^{6}\right\rangle /$ $\left\langle r^{5}\right\rangle \cong\langle r\rangle$. This simple approximation becomes however increasingly inaccurate when the degree of polydispersity is beyond $\sim 15 \%$. Furthermore, while at first sight DDLS might seem only as a trivial extension of DLS, there are distinct aspects. First of all, DDLS in fact enables more elaborate and advanced analyses of particle systems because DDLS is able to resolve size distributions better than DLS. To understand this, let us consider a small change in the radius $(r+\mathrm{d} r)$ that will change both the rotational $\left(D_{\mathrm{R}}+\Delta D_{\mathrm{R}}\right)$ and the translational $\left(D_{\mathrm{T}}+\Delta D_{\mathrm{T}}\right)$ diffusion coefficients. Let $\mathrm{d} r=r+\varepsilon r$, where $\varepsilon$ is a nonzero real number $(|\varepsilon|>0)$, and it is easy to show through a series expansion that even when the change is very small (that is, $|\varepsilon| \ll 1),\left|\Delta D_{\mathrm{R}} / D_{\mathrm{R}}\right|=3 \varepsilon$ while $\left|\Delta D_{\mathrm{T}} / D_{\mathrm{T}}\right|=\varepsilon$. This means that compared to DLS, DDLS has a three times stronger response towards changes in the particle size. Consequently, owing to rotational diffusion, DDLS is capable of resolving differences in particle sizes more effectively than DLS, due to the $r^{-3}$ dependence of $D_{\mathrm{R}}$ dominating the relaxation rate of the DDLS spectra of NPs. When the NPs are polydisperse, the rotational and translational diffusion coefficients are also dispersed. It follows from this that a standard unimodal distribution in $P(\Gamma)$ is usually unfit to describe DDLS spectra as soon as the number weighted polydispersity is beyond $30 \% .^{32}$ Actually, this is the very reason why depolarized field auto-correlation functions from polydisperse samples appear as strongly stretched exponential functions. ${ }^{3,34} \mathrm{~A}$ detailed discussion addressing the impact of polydispersity on the
DDLS spectrum is given in the $\mathrm{ESI} \dagger$ (Influence of polydispersity on DDLS spectra), and to demonstrate our polydispersity analysis, we use aqueous suspensions of gold nanoparticles (Au NPs) of different sizes and surface functionalization. On the one hand, Au NPs have been receiving increasing attention due to their unique size-dependent properties ${ }^{35,36}$ and on the other hand, they possess intrinsic imperfections that result in optical anisotropy. ${ }^{32,37,38}$ Yet, these imperfections are small enough to preserve a rather high degree of spherical symmetry, which enables the applicability of the spherical model.

\section{Results and discussion}

\section{Citrate-coated gold nanoparticles}

Firstly, four different batches of citrate-coated Au NPs were synthesized (Au1, Au2, Au3, Au4). Their transmission electron microscopy (TEM) micrographs are shown in Fig. 1 and the results of the image analyses are summarized in Table 1.

Next, both depolarized (DDLS) and polarized (DLS) light scattering spectra were recorded from dilute aqueous suspensions of the $\mathrm{Au}$ NPs (conc. $\sim 20 \mu \mathrm{g} \mathrm{mL}^{-1}$ ). Many parametric statistical distributions have been applied to describe particle systems, ${ }^{39}$ and here we adopt the lognormal distribution to analyse the DDLS spectra. In this case, the $j^{\text {th }}$ raw moment of $P(r)$ can be written as a function of the average particle size $r$ and the particle polydispersity index $\sigma$ defined as the standard deviation divided by the mean (ESI, $\uparrow$ The lognormal distribution):

$$
\left\langle r^{j}\right\rangle=\langle r\rangle^{j}\left(1+\sigma^{2}\right)^{\frac{1}{2}(j-1) j} .
$$

By fitting the experimental data against a standard polynomial (Fig. 2), the coefficients corresponding to the ratios of the raw moments (eqn (2)) are determined. The average particle size $\langle r\rangle$ and the particle polydispersity index $\sigma$ are determined from the ratios of the raw moments, using eqn (3). The DLS spectra of optically anisotropic NPs have two modes (Fig. 2): one mode corresponds to the translational motion, and the other one does to both rotational and translational motions. ${ }^{28}$ Therefore, the DLS spectra were fitted against a bimodal function, where each mode is represented by a stretched exponential:

$$
g_{1, \mathrm{DLS}}(t)=y e^{-\left(\Gamma_{1} t\right)^{\nu_{1}}}+(1-y) e^{-\left(\Gamma_{2} t\right)^{\nu_{2}}},
$$

where $\Gamma_{1} \equiv q^{2} D_{\mathrm{T}}, \Gamma_{2} \equiv 6 D_{\mathrm{R}}+q^{2} D_{\mathrm{T}}, 0<v_{1}, v_{2}<1$, and $0<y<1$.

DLS determines the $Z$-average equal to $\left\langle r^{6}\right\rangle /\left\langle r^{5}\right\rangle$. To compare the DDLS results with DLS, $Z$-averages were also calculated from $P(r)$ via the lognormal model: $\left\langle r^{6}\right\rangle /\left\langle r^{5}\right\rangle=\langle r\rangle\left(1+\sigma^{2}\right)^{5}$. In the case of DLS, the $Z$-average was directly quantified by fitting the DLS spectra against the bimodal function, and then using the slower mode of the fit. This mode corresponds to translational diffusion. The results are summarized in Table 2. The agreement between DDLS and DLS is very good. We point out that the $Z$-average of polydisperse particles is always greater 

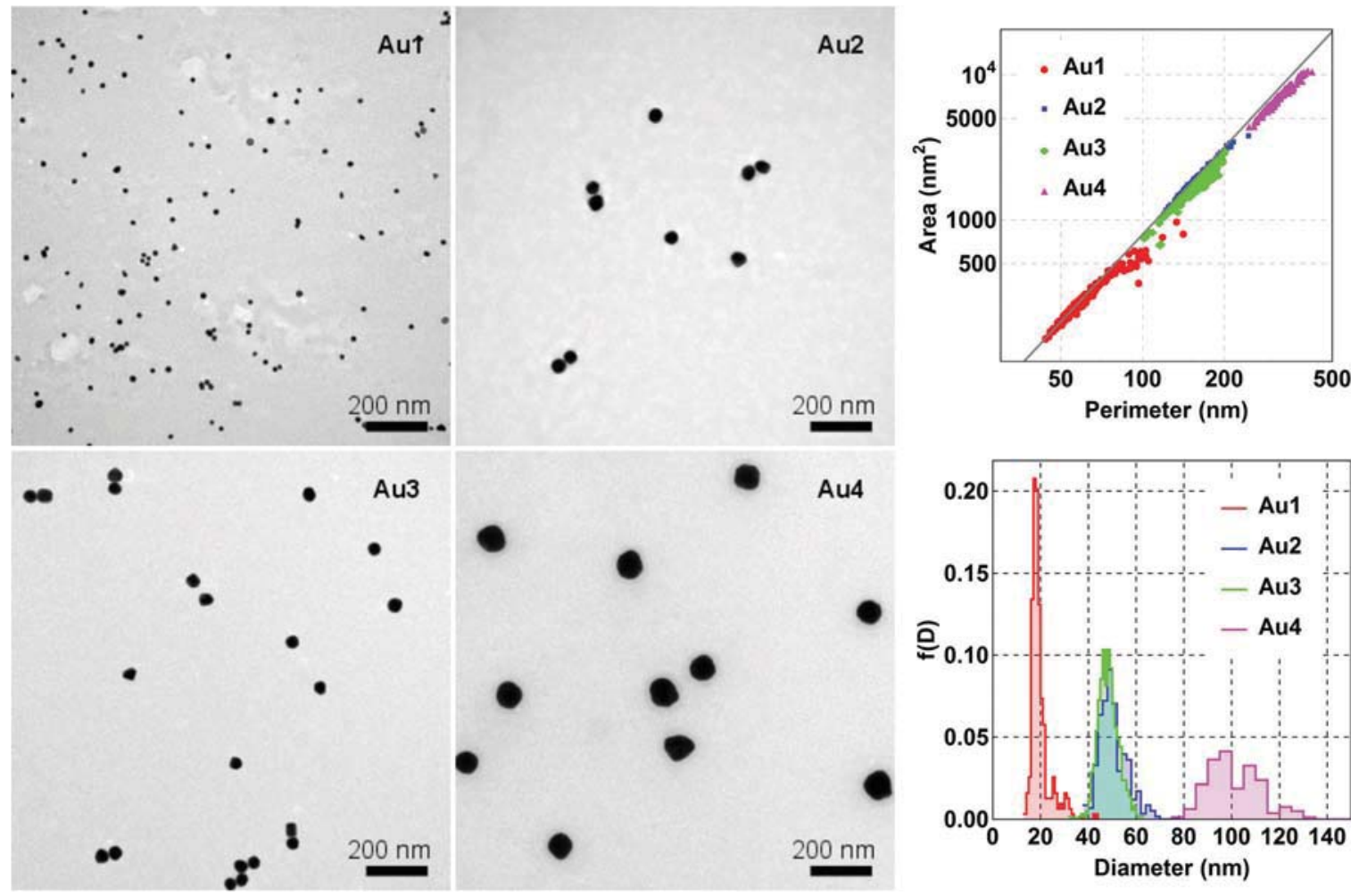

Fig. 1 Transmission electron microscopy (TEM) micrographs of the Au NPs and the results of the image analysis. Right top: the area (A) versus the perimeter $(P)$ of the particles. The solid line, $P^{2} / 4 \pi$, indicates the relationship for perfect spheres. Data falling below this line indicates deviations from a sphere. Deviation from the spherical shape may be quantified by the circularity: $\sqrt{4 \pi A / P^{2}}$. Right bottom: the estimated probability density distribution of the diameter $(D) . f(d) \times \mathrm{d} D$ quantifies the probability that the diameter is found in $\mathrm{d} D$ interval about $D$. The diameter of a particle is evaluated from its perimeter: $D=P / \pi$. This value corresponds to the diameter of a perfect sphere of the same perimeter, and it can be shown that for a convex shape, it is the Feret diameter averaged over all possible particle orientation. ${ }^{39}$

Table 1 Summary of TEM analyses of citrate-coated Au NPs

\begin{tabular}{lccc}
\hline & Au1 & Au2 & Au3 \\
\hline Feret diameter (mean \pm std/mean) & $20 \mathrm{~nm} \pm 21 \%$ & $51 \mathrm{~nm} \pm 12 \%$ & $48 \mathrm{~nm} \pm 9 \%$ \\
Circularity (mean \pm std/mean) & $0.98 \pm 5 \%$ & $0.97 \pm 2 \%$ & $0.95 \pm 2 \%$
\end{tabular}
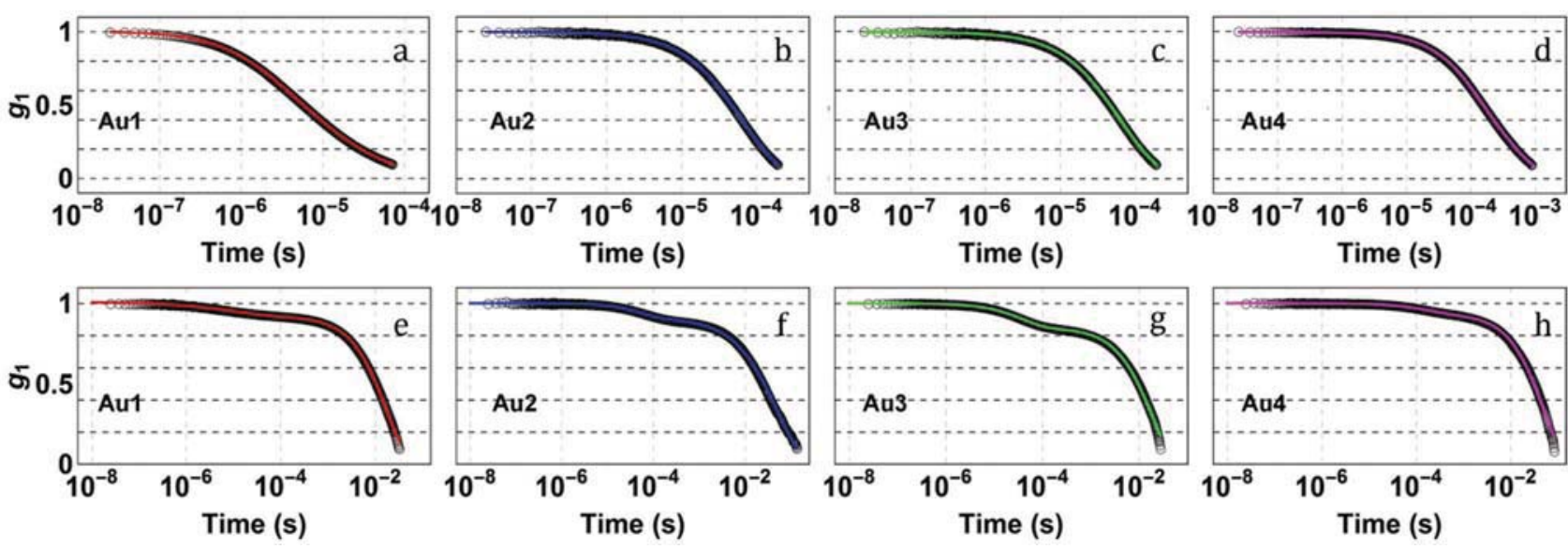

Time (s)

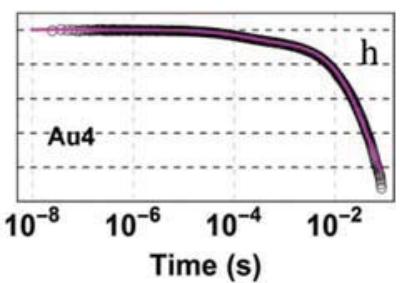

Fig. 2 The DDLS ( $\mathrm{a}-\mathrm{d}$, top) and DLS (e-h, bottom) field auto-correlation functions $\left(\theta=10^{\circ}\right)$ corresponding to the four different citrate-coated Au NPs (Au1-Au4, from left to right). The solid lines are the best fits (DDLS and DLS, eqn (2) and (4) respectively). 
Table 2 Summary of DDLS and DLS analyses of the citrate-coated Au NPs

\begin{tabular}{llll}
\hline Hydrodynamic diameter & Au1 & Au2 & Au3 \\
\hline DDLS lognormal model (mean \pm std/mean) & $23 \mathrm{~nm} \pm 32 \%$ & $50 \mathrm{~nm} \pm 35 \%$ & $52 \mathrm{~nm} \pm 21 \%$ \\
DDLS Z-average via lognormal model & $37 \mathrm{~nm}$ & $89 \mathrm{~nm}$ & $64 \mathrm{~nm}$ \\
DLS Z-average & $38 \mathrm{~nm}$ & $92 \mathrm{~nm}$ & $101 \mathrm{~nm} \pm 18 \%$ \\
& & $122 \mathrm{~nm}$ & $66 \mathrm{~nm}$ \\
\hline
\end{tabular}

than the number-averaged mean: $\left\langle r^{6}\right\rangle /\left\langle r^{5}\right\rangle>\langle r\rangle \quad$ ESI, $\dagger$ $Z$-average). The consistency between DDLS and TEM is good. Nevertheless, compared to the perimeter-equivalent size determined from TEM (Table 1), DDLS analyses using the lognormal model tends to determine larger values for size polydispersity. This difference may result from several phenomena that are discussed in the ESI† (TEM versus light scattering).

To test the results provided by DDLS, next we characterized the aqueous suspensions of the Au1 and Au2 NPs by using two well-established in situ techniques that also probe hydrodynamic size and are commercially available: nanoparticle tracking analysis (NTA) and Taylor-Aris dispersion (TDA). Here we only summarize the results and all details are given in the ESI $\dagger$ (Nanoparticle tracking analysis and Taylor-Aris dispersion). Using NTA with the performance available, the instrument was unable to track the small NPs (Au1 NPs). Considering the characterization of the Au2 NPs, NTA analysis determined larger particles and a wider distribution (mean diameter: $81 \mathrm{~nm}$, polydispersity index: $62 \%$ ) but the overall tendency is not entirely inconsistent when compared to TEM and DDLS (Fig. S2 $\dagger$ ). The discrepancy most likely owes to the fact that the sensitivity of NTA at the lower end of the size range is cut off. ${ }^{40}$ This cutoff begins at $\sim 30 \mathrm{~nm}$ in the case of Au NPs, and thus, one obtains a size distribution that is skewed towards larger sizes.

Not having this kind of limitation, TDA spectra obtained at two different detection windows $(45 \mathrm{~cm}$ and $85 \mathrm{~cm}$, ESI, $\uparrow$ Taylor-Aris dispersion) are in excellent agreement with light scattering (Table 3). The apparent hydrodynamic radius of the Au NPs determined by TDA is equal to $\left\langle r^{4}\right\rangle /\left\langle r^{3}\right\rangle$ (ESI, $\dagger$ TaylorAris dispersion). In the case of DDLS, this value can be calculated via the lognormal model (eqn (3) and Table 2): $\left\langle r^{4}\right\rangle /\left\langle r^{3}\right\rangle=$ $\langle r\rangle\left(1+\sigma^{2}\right)^{3}$.

To test further our DDLS approach, we recorded and analysed correlation functions from a bimodal dispersion prepared by mixing the Au1 and Au2 NPs. In the case of bimodal suspensions, the mean relaxation rate is the intensity-weighted sum of the relaxation rates of the respective

Table 3 Characterization of citrate-coated Au NPs by TDA

\begin{tabular}{lll}
\hline Hydrodynamic diameter & Au1 & Au2 \\
\hline TDA (det. win. @45 cm) & $33 \mathrm{~nm}$ & $64 \mathrm{~nm}$ \\
TDA (det. win. @ $85 \mathrm{~cm}$ ) & $31 \mathrm{~nm}$ & $60 \mathrm{~nm}$ \\
DDLS lognormal model $\left\langle r^{4}\right\rangle /\left\langle r^{3}\right\rangle$ & $33 \mathrm{~nm}$ & $71 \mathrm{~nm}$
\end{tabular}

modes (ESI, $\uparrow$ Depolarized scattering from multimodal suspensions):

$$
\begin{aligned}
\langle\Gamma\rangle= & A_{1} \frac{k_{\mathrm{B}} T}{\pi \eta}\left(\frac{6}{8} \frac{\left\langle r_{1}{ }^{3}\right\rangle}{\left\langle r_{1}{ }^{6}\right\rangle}+\frac{q^{2}}{6} \frac{\left\langle r_{1}{ }^{5}\right\rangle}{\left\langle r_{1}{ }^{6}\right\rangle}\right) \\
& +A_{2} \frac{k_{\mathrm{B}} T}{\pi \eta}\left(\frac{6}{8} \frac{\left\langle r_{2}{ }^{3}\right\rangle}{\left\langle r_{2}{ }^{6}\right\rangle}+\frac{q^{2}}{6} \frac{\left\langle r_{2}{ }^{5}\right\rangle}{\left\langle r_{2}{ }^{6}\right\rangle}\right) .
\end{aligned}
$$

Given that at an equal mass-based concentration the Au2 NPs scatter approximately five times more than the Au1 NPs (Fig. 3a), we prepared a vol. (Au1): vol. (Au2) $=5: 1$ bimodal suspension (Fig. 3b). The relaxation rate $\langle\Gamma\rangle$ as a function of $q^{2}$ is shown in Fig. 3c. Since $A_{1}<1$ and $A_{2}<1$ the value of $\langle\Gamma\rangle$ is expected to be between the relaxation rates of the respective modes, i.e. $\left\langle\Gamma_{1}\right\rangle \leq\langle\Gamma\rangle \leq\left\langle\Gamma_{2}\right\rangle$ when $\left\langle\Gamma_{1}\right\rangle\left\langle\left\langle\Gamma_{2}\right\rangle\right.$. Indeed this is the case, as shown by the curves predicted by eqn (5), without involving either adjustable or free parameters. The experimental result and the theoretical prediction agree very well with one another, which shows that rotational diffusion is way more sensitive to bimodality than translational diffusion: the slope of the line hardly changes compared to the intercept.

\section{PEG-coated gold nanoparticles (PEG-CH 3 Au NPs)}

Au NPs often carry a synthetic polymer coating that either promotes colloidal stability or allows further functionalization. ${ }^{41}$ On the account of such functionalities, interactions with natural biopolymers, ${ }^{19,42,43}$ salts, vitamins, and lipids ${ }^{44}$ found abundantly in biological and physiological fluids, can be either suppressed ${ }^{45}$ or enhanced. ${ }^{13}$ These interactions can strongly influence the composition and formation of the socalled protein corona, ${ }^{11}$ which can control the cellular response $^{46}$ and biological fate. ${ }^{13,47}$ Additionally, the presence of a polymeric shell will alter the diffusion and sedimentation rates, ${ }^{48}$ which are particularly relevant for in vitro nanoparticle dosimetry. ${ }^{15,49}$ Next we show that the combination of DDLS with other techniques, such as small-angle X-ray scattering (SAXS) and TEM, accurately characterizes the polymer shell thickness. In a protein-rich environment obtaining satisfactory micrographs via standard TEM is difficult; however, soft X-rays can provide sufficient scattering contrast in favour of the inorganic core, and DDLS has clear advantages when characterizing the polymer coat in biological/physiological media. We used another batch of Au NPs (size $15 \mathrm{~nm}$, Fig. S6†) coated with end-thiolated poly(ethylene glycol) (PEG), where the thiolated PEG had a methoxy $\left(-\mathrm{OCH}_{3}\right)$ end group. SAXS and TEM (ESI, $\uparrow$ Small-angle X-ray scattering and TEM of PEG-coated Au NPs) were used to measure the size of the gold core, while DDLS analysis was used to determine the hydrodynamic size 


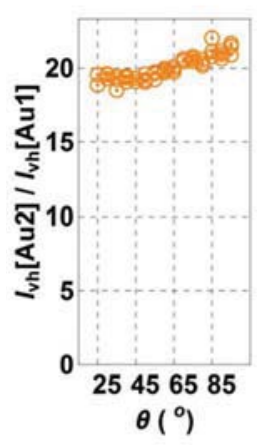

b

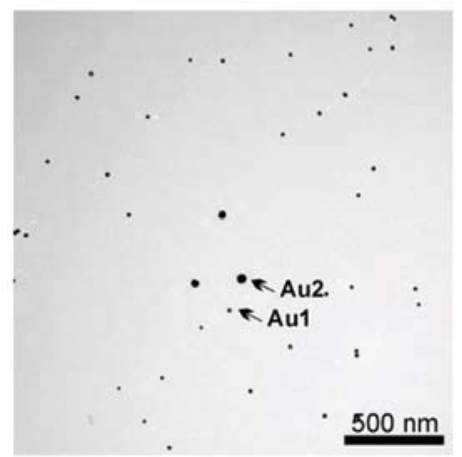

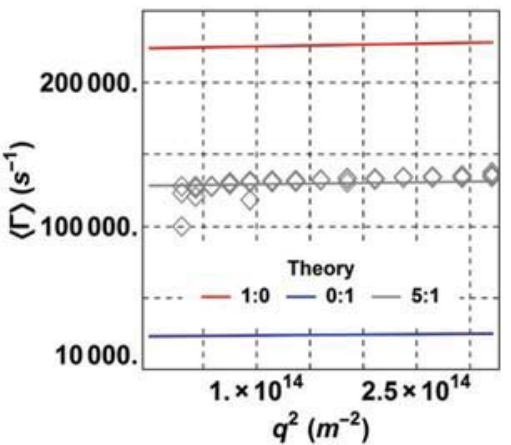

Fig. 3 (a) The ratio of depolarized scattering from the Au1 and Au2 NPs measured as a function of the scattering angle. (b) The TEM micrograph of the bimodal suspension obtained by mixing the Au1 and Au2 NPs. (c) The relaxation rate $\langle\Gamma\rangle$ as a function of $q^{2}$. The solid lines are relaxation rates predicted by theory: $1: 0 \rightarrow 1 \times$ vol. (Au1) : $0 \times$ vol. (Au2), $0: 1 \rightarrow 0 \times$ vol. (Au1) : $0 \times$ vol. (Au2), and $5: 1 \rightarrow 5 \times$ vol. (Au1) : $1 \times$ vol. (Au2). The symbol stands for the experimental result obtained with the bimodal dispersion.

defined by the polymer enclosing the core. Fitting the SAXS spectrum against a spherical model resulted in a narrow core size distribution (polydispersity index 13\%), and an average diameter of $13.5 \mathrm{~nm}$. TEM characterization of these NPs resulted in similar values: $16 \mathrm{~nm}$ and $16 \%$ polydispersity. The relative difference between the result of SAXS and TEM is not high $(<8 \%)$, and can be attributed to the fact that the gold core is actually slightly spheroidal (ESI, Fig. S6b $\dagger$ ). In the presence of a polymer grafted or adsorbed onto the surface, we can express the hydrodynamic radius as a function of the core radius: $r_{\mathrm{H}}=\gamma(r) \times r$, where $\gamma(r)>1 . \gamma(r)$ is a function that can embody properties related to the polymer, such as molecular weight, graft density, surface coverage and conformation. For polymer coated particles, we can adapt a modified version of eqn (2):

$$
\begin{aligned}
g_{1}(t)=1 & -\frac{k_{\mathrm{B}} T}{\pi \eta}\left(\frac{6}{8} \frac{\left\langle r^{3}\right\rangle}{\gamma^{3}\left\langle r^{6}\right\rangle}+\frac{q^{2}}{6} \frac{\left\langle r^{5}\right\rangle}{\gamma\left\langle r^{6}\right\rangle}\right) t \\
& +\left(\frac{k_{\mathrm{B}} T}{\pi \eta}\right)^{2}\left(\frac{9}{32} \frac{1}{\left\langle r^{6}\right\rangle}+\frac{q^{2}}{8} \frac{\left\langle r^{2}\right\rangle}{\gamma^{4}\left\langle r^{6}\right\rangle}+\frac{q^{4}}{72} \frac{\left\langle r^{4}\right\rangle}{\gamma^{2}\left\langle r^{6}\right\rangle}\right) t^{2} \\
& +\ldots,
\end{aligned}
$$

where $\gamma$ represents the factor quantifying the hydrodynamic radius of the polymer shell. The derivation of eqn (6) is given in the ESI $\dagger$ (Correlation function of depolarized scattering from polymer-coated particles). Via the lognormal model (Fig. 4a), a mean diameter of $2 r_{\mathrm{H}}=40 \mathrm{~nm}(\gamma \cong 3)$ and a polydispersity index of $19 \%$ were determined by DDLS analysis. This means in an average shell thickness of $\sim 13 \mathrm{~nm}$, in agreement with previous reports, ${ }^{50,51}$ which is reasonable given an upper limit as the polymer contour length, $35 \mathrm{~nm}$, and the Flory radius as a lower limit, $3 \mathrm{~nm} .{ }^{32}$ To compare DDLS against DLS (Fig. 4b), Z-averages were obtained in the same way as explained in the case of the Au1-4 NPs. DLS estimates $46 \mathrm{~nm}$, and DDLS $48 \mathrm{~nm}$. This agreement is very good for the relative difference is within $5 \%$.
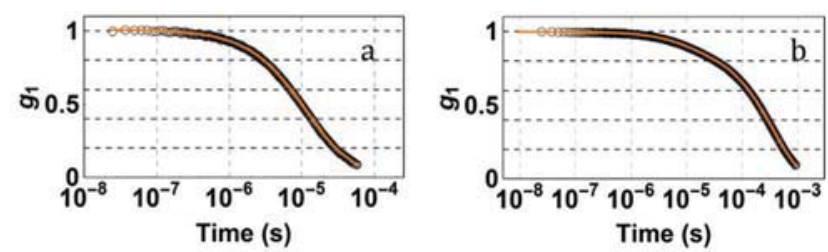

Fig. 4 The DDLS (a) and DLS (b) field auto-correlation functions $(\theta=$ $15^{\circ}$ ) corresponding to the PEG-coated gold citrate NPs. The solid lines are the best fits.

To test further the accuracy of our DDLS analysis, we recorded additional DDLS correlation functions at higher angles $\left(\theta=20^{\circ}-135^{\circ}\right)$. It follows from eqn (1) that the values of the statistical moments of $P(\Gamma)$ are absolutely independent of the model applied - regardless of shape and distribution and can be determined experimentally directly from $g_{1}$. Based on the best fit (Fig. 4a) we have simulated the first three raw moments of $P(\Gamma)$ at different angles, without involving either free or adjustable parameters (ESI, $\dagger$ Raw moments of $P(\Gamma)$ ). The data points corresponding to $\langle\Gamma\rangle$ directly relate to $6 D_{\mathrm{R}}$ and $D_{\mathrm{T}}$, respectively. The data points corresponding to $\left\langle\Gamma^{2}\right\rangle$ relate to the width of the distribution, and the data points corresponding to $\left\langle\Gamma^{3}\right\rangle$ to the symmetry/asymmetry of the distribution (skewness) with respect to the mean. Fig. 5 shows that these three moments of $\Gamma$ span are over about ten orders of magnitude. Yet, the agreement between experimental data and our simulation is excellent, which indicates that the characterization of the size distribution is good.

\section{Citrate-coated gold nanoparticles incubated in BSA solution}

In order to show that the here-presented approach is also helpful for characterizing the formation of the protein corona in complex physiological/biological media, two Au NP suspensions (Au1 and Au2) were incubated for 16 hours with bovine 


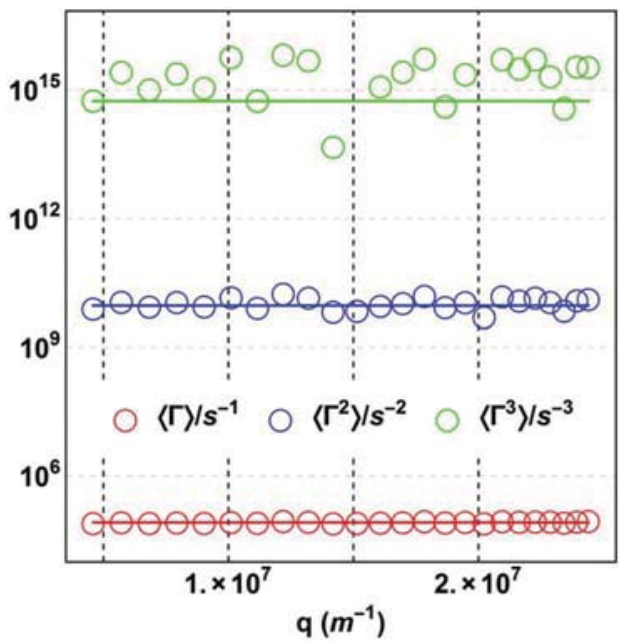

Fig. 5 The first three raw moments of $P(\Gamma)$ as functions of $q\left(\theta=20^{\circ}\right.$ $\left.135^{\circ}\right)$. For each $g_{1}(t, \theta),\left\langle\Gamma^{n}\right\rangle$ is simultaneously but independently quantified through experiments and theory without involving either adjustable or free parameters (ESI, $\uparrow$ Raw moments of $P(\Gamma))$. The symbols are experimental values and the solid lines are obtained via the lognormal and spherical models whose parameters $\langle r\rangle, \sigma$, and $\gamma$ were estimated from a single $g_{1}(t)$ measured at another angle.

serum albumin (BSA). Fig. 6 shows that - as expected - BSA was adsorbed onto the particle surface. Owing to the increased hydrodynamic size, the corresponding correlation function decays slowly, and no sign of aggregation was observed. The number-averaged mean particle size and polydispersity index were estimated as above. In the case of the Au1 NPs, the average hydrodynamic diameter has increased from $23 \mathrm{~nm}$ to $45 \mathrm{~nm}(45 \mathrm{~nm} \pm 32 \%$, mean $\pm \mathrm{std} /$ mean $)$, and in the case of the Au2 NPs, $50 \mathrm{~nm}$ to $80 \mathrm{~nm}(80 \mathrm{~nm} \pm 22 \%)$. Therefore, the incubation and the excess of protein resulted in a relatively thick BSA shell with an average thickness of more than $10 \mathrm{~nm}$, although addressing the fine details of the formation of the BSA layer is beyond the scope of this work..$^{52,53}$ The presence of a BSA shell formed by adsorption onto the particle surfaces is clearly indicated also by UV-vis analysis, ${ }^{37}$ for a red-shift in the centre position of the LSPR spectra can be observed (ESI, $\uparrow$ UV-vis spectra of gold citrate NPs incubated in BSA solution, Fig. S7†).

While DDLS enables the suppression of light scattering from the constituents of complex biological and physiological media, ${ }^{37}$ the interpretation may easily become difficult with DLS. One must take into account at least three modes: one mode for the translational motion, the second for both rotational and translational motions, and one must include at least one more additional mode, to account for the protein rich media (ESI, $\uparrow$ Light scattering from nanoparticles in biological media). By using a tri-modal approach we could not find any satisfactory agreement between the DLS model and the experimental data. It is likely that additional modes are required. Therefore, while on paper it is straightforward, DLS in complex fluids is not a viable option in practice, given the increasing number of free parameters required for data fitting.

\section{Conclusions}

The analysis presented here determined accurately the number-averaged size distribution of Au NPs of different size and surface functionalization. The analysis using the raw moments is universally valid as long as the scattering intensity is proportional to the square of the particle volume. This condition is satisfactory for many nanoparticle systems of current interest. Given that no particle is really homogeneous with a perfect central symmetry, there are many particle systems that depolarize light, such as Janus or porous particles. Furthermore, the analysis can be extended for particle systems other than spherical particles, including spheroids, ${ }^{54}$ nanorods, ${ }^{55-57}$ and aggregates. ${ }^{49,58}$

\section{Materials and experimental methods}

\section{Citrate-coated AuNPs}

The Au1 particles were synthesized via reduction of $\mathrm{Au}^{3+}$, following the procedure of Grabar, et al. ${ }^{59}$ Briefly, the vigorously
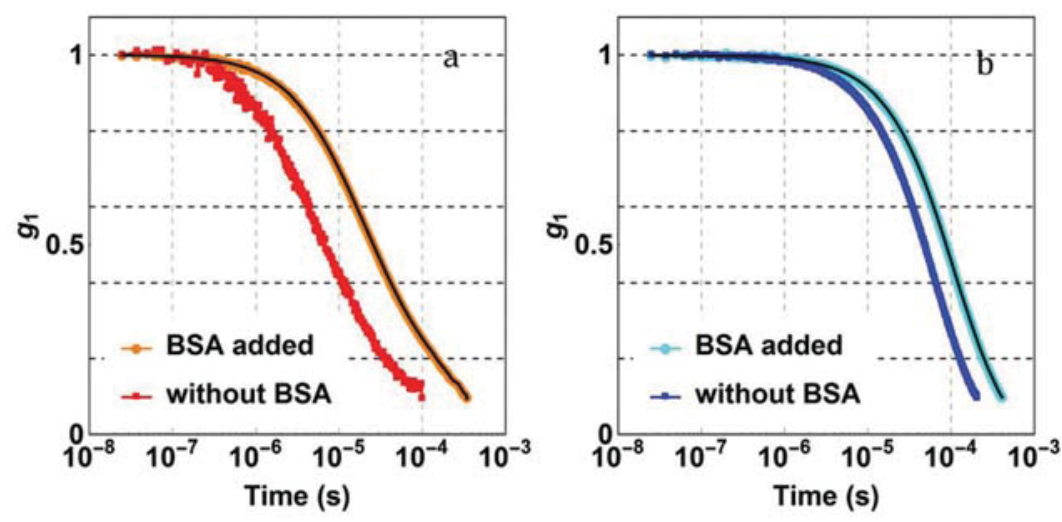

Fig. 6 Field auto-correlation functions of Au1 (a) and Au2 (b) particles $\left(\theta=15^{\circ}\right)$ with and without BSA. The solid lines are the best polynomial fits. 
stirred aqueous tetrachloroauric acid solution $(125 \mathrm{~mL}$, $0.25 \mathrm{mM} \mathrm{HAuCl}{ }_{4} \cdot 3 \mathrm{H}_{2} \mathrm{O}$, Sigma-Aldrich) was brought to boil, which was followed by the rapid addition of $12.5 \mathrm{~mL}$ of $40 \mathrm{mM}$ sodium citrate to the vortexed $\mathrm{HAuCl}_{4}$ solution. The solution was maintained at boiling temperature for another 15 minutes and then removed from heat, while stirring continuously for an additional 15 minutes.

The Au2 particles were synthesized by the overgrowth of gold onto the Au1 particles (seed dispersion) as described by Brown, et $a{ }^{60}{ }^{60}$ The growth step was carried out by adding hydroxylamine-hydrochloride $\left(\mathrm{NH}_{2} \mathrm{OH} \cdot \mathrm{HCl} ; 3 \mathrm{~mL}\right.$ of $0.2 \mathrm{M}$; Sigma-Aldrich) and Au1 NPs (15 mL) to a solution of $\mathrm{HAuCl}_{4}$ $(0.25 \mathrm{mM}$ in $270 \mathrm{~mL})$. The resulting gold particles were subsequently functionalized with sodium citrate $(1.7 \mathrm{~mL}$ of $40 \mathrm{mM}$ sodium citrate), washed by centrifugation at $3500 \mathrm{rpm}$ for 30 minutes, and finally re-dispersed in Millipore water.

Both the Au3 and Au4 particles were synthesized by the overgrowth of gold onto small 'seed' gold particles, which were synthesized as reported by Turkevich et al. ${ }^{61}$ These Au seed suspensions $(d=15 \mathrm{~nm},[\mathrm{Au}]=0.5 \mathrm{mM})$ were used subsequently to grow larger citrate stabilized $\mathrm{Au}$ particles. ${ }^{60} \mathrm{An}$ aqueous solution of $\mathrm{HAuCl}_{4}$ and hydroxylamine-hydrochloride was prepared in a glass bottle, and homogenized with a magnetic stirrer. The gold seed suspension was added and the mixture was stirred constantly for approximately $20 \mathrm{~min}$. This resulted in Au3 NPs. A second growth step was continued using the same procedure as the first growth step, with the exception of using the freshly prepared Au3 particles as the seed solution. This resulted in Au4 particles.

\section{PEG-CH $_{3}$ Au NPs}

First, citrate-stabilized $\mathrm{Au}$ NPs $([\mathrm{Au}]=0.5 \mathrm{mM})$ were synthesized as reported by Turkevich et al. ${ }^{61}$ Aqueous solutions $\left(3.4 \times 10^{-3} \mathrm{mM}\right)$ of thiolated methoxy-poly(ethylene glycol) $\left(\mathrm{PEG}-\mathrm{CH}_{3}, M_{\mathrm{W}}=5000 \mathrm{~g} \mathrm{~mol}^{-1}\right)$ were sonicated for $15 \mathrm{~min}$, subsequently mixed with $100 \mathrm{~mL}$ of the $\mathrm{Au} \mathrm{NP}$ suspension and left to react at $25{ }^{\circ} \mathrm{C}$ for $24 \mathrm{~h}$. This mixing ratio is expected to provide approximately 10 molecules of the polymer for each $\mathrm{nm}^{2}$ of the particle surface. The PEGylated particles were centrifuged twice for $1 \mathrm{~h}$ at $10^{4} \mathrm{~g}$ to remove the excess polymer, and re-dispersed in $10 \mathrm{~mL}$ of water. Finally, the particles were transferred to a phosphate buffer (PBS, $10 \mathrm{mM}$ sodium phosphate monobasic/disodium phosphate hydrogen, $\mathrm{pH} 7$ ).

\section{Characterization}

For TEM, $50 \mu \mathrm{g} \mathrm{mL}^{-1} \mathrm{Au}$ NP suspensions (Au1 and Au2) were incubated with $5 \mathrm{mg} \mathrm{mL} \mathrm{mL}^{-1}$ bovine serum albumin (BSA), where $40 \mu \mathrm{L}$ of $130 \mathrm{mg} \mathrm{mL}^{-1}$ BSA solution was added to $1 \mathrm{~mL}$ of Au NP suspension and left for $16 \mathrm{~h}$. Micrographs of the Au NPs were obtained with a Tecnai Spirit transmission electron microscope (FEI), operating at $120 \mathrm{kV}$. The images were recorded at a resolution of $2048 \times 2048$ pixels (Veleta CCD camera, Olympus). The sample preparation followed the protocol presented in ref. 62: to prevent the formation of drying artefacts, the colloidal suspension was mixed with an aqueous solution of bovine serum albumin (BSA), and $5 \mu \mathrm{l}$ of this mixture was drop cast onto a carbon-film square mesh copper grid (Electron Microscopy Sciences, CF-300-Cu) and dried in ambient air. The micrographs of the Au NPs were analysed via image processing (ImageJ, National Institutes of Health, USA).

Light scattering data were collected at a constant temperature $\left(22^{\circ} \mathrm{C}\right)$ using a commercial goniometer (3D LS Spectrometer, LS Instruments AG, Switzerland). The primary beam was formed by a linearly polarized and collimated laser beam (Cobolt 05-01 diode pumped solid state laser, $\lambda=660 \mathrm{~nm}, P_{\text {max. }}$. $=500 \mathrm{~mW}$, and HeNe laser, $\lambda=632.8 \mathrm{~nm}, P_{\max }=21 \mathrm{~mW}$ ), and the scattered light was collected by single-mode optical fibres equipped with integrated collimation optics. The collected light was coupled into two high-sensitivity APD detectors via laser-line filters (Perkin Elmer, Single Photon Counting Module), and their outputs were fed into a two-channel multiple-tau correlator. The signal-to-noise ratio was improved by cross-correlating these two channels. With respect to the primary beam, depolarized scattering was observed via crosspolarizers. The incoming laser beam was passed through a Glan-Thompson polarizer with an extinction ratio of $10^{-6}$, and another Glan-Thompson polarizer, with an extinction ratio of $10^{-8}$, was mounted in front of the collection optics. Count rates varied, typically between 50 and $500 \mathrm{kHz}$, depending on the particle system and angle of scattering. The average photon count rate from the dark noise of our APD detector is not more than $0.5-0.6 \mathrm{kHz}$. Therefore, as long as the count rate is sufficiently high (e.g. $>30 \mathrm{kHz})$ - compared to the detector dark noise - it does not influence the quality of the correlation function. The field auto-correlation functions were obtained via the Siegert relation: $g_{1}(t)=\sqrt{g_{2}(t)-1}$ where $g_{2}(t)$ is the intensity auto-correlation function constructed from the temporal fluctuations of the depolarized component of the scattered intensity. Regarding fitting, it is worthwhile to point out that $g_{1}(t)$ is always expected to be a monotonic decreasing function of time, since $\partial_{t} g_{1}(t)<0$. Furthermore, $g_{1}(t)$ is also expected to be a convex function, since $\partial_{t}^{2} g_{1}(t)>0$. A sequence of correlation data that do not satisfy these general properties are non-physical and represent artefacts, and thus, ideally should be discarded and not analysed. The residuals between fit and experimental data should not exhibit any trend as a function of time, and their distribution should follow a normal distribution with zero mean.

Small-angle X-ray scattering (SAXS) spectrum was recorded by using a NanoMax-IQ camera (Rigaku Innovative Technologies, Auburn Hills, MI USA). The aqueous suspension was kept in a $1 \mathrm{~mm}$ capillary at room temperature during the measurements. Raw data were processed according to standard procedures, and the scattering spectra are presented as a function of the momentum transfer $q=4 \pi \lambda^{-1} \sin (\theta / 2)$, where $\theta$ is the scattering angle and $\lambda=0.1524 \mathrm{~nm}$ is the photon wavelength.

UV-vis spectra of the samples were recorded at $25^{\circ} \mathrm{C}$ using a Jasco V-670 spectrophotometer and a $10 \mathrm{~mm}$-path-length quartz cuvette.

Taylor-Aris dispersion (TDA) spectra were obtained by using an ActiPix D100 UV area imaging detector operating at a 
sampling rate of $20 \mathrm{~Hz}$ (Paraytec, York, UK) equipped with a bandpass filter $(520 \mathrm{~nm})$ and a neutral density filter of $10 \%$ transmission. The particle suspensions were injected into a fused silica capillary (74.5 $\mu \mathrm{m}$ inner diameter, Polymicro Technologies) under a steady laminar flow, using a capillary electrophoresis injection system (Prince 560 CE Autosampler, Prince Technologies B.V., Netherlands). After sample injection (160 mbar for $6 \mathrm{~s}$ ), a pressure of 40 mbar was applied to transport the NP samples through the capillary at a constant temperature of $25^{\circ} \mathrm{C}$. A $0.001 \%(\mathrm{wt} / \mathrm{v}$ ) TWEEN-20 solution was used as the running buffer. The total capillary length was $130 \mathrm{~cm}$, and the centers of the two detection windows (width of $10 \mathrm{~mm}$ ) were at $45 \mathrm{~cm}$ and $85 \mathrm{~cm}$.

\section{Author contributions}

The manuscript was written through contributions of all authors. All authors have given approval to the final version of the manuscript.

\section{Acknowledgements}

The authors are grateful for the financial support of the Adolphe Merkle Foundation and the University of Fribourg. We acknowledge the financial support of the Swiss National Science Foundation (through the National Centre of Competence in Research Bio-Inspired Materials (SB), the National Research Programme "Resource Wood" (CG) and Grant \# PP00P2126483/1 (AP-F). L. Rodriguez-Lorenzo acknowledges the financial support from the L'Oreal Switzerland and UNESCO's fellowship program "For Women in Science 2013".

\section{References}

1 T. Tsuzuki, Int. J. Nanotechnol., 2009, 6, 567-578.

2 A. Z. Moshfegh, J. Phys. D: Appl. Phys., 2009, 42, 233001.

3 B. E. Hayden, Acc. Chem. Res., 2013, 46, 1858-1866.

4 M. Gonzales-Weimuller, M. Zeisberger and K. M. Krishnan, J. Magn. Magn. Mater., 2009, 321, 1947-1950.

5 N. G. Khlebtsov and L. A. Dykman, J. Quant. Spectrosc. Radiat. Transfer, 2010, 111, 1-35.

6 A. E. Ershov, I. L. Isaev, P. N. Semina, V. A. Markel and S. V. Karpov, Phys. Rev. B: Condens. Matter, 2012, 85, 045421.

7 C. L. Phillips, C. R. Iacovella and S. C. Glotzer, Soft Matter, 2010, 6, 1693-1703.

8 R. J. Macfarlane, B. Lee, M. R. Jones, N. Harris, G. C. Schatz and C. A. Mirkin, Science, 2011, 334, 204-208.

9 G. Bushell and R. Amal, J. Colloid Interface Sci., 1998, 205, 459-469.

10 M. L. Eggersdorfer and S. E. Pratsinis, Aerosol Sci. Technol., 2011, 46, 347-353.

11 A. E. Nel, L. Madler, D. Velegol, T. Xia, E. M. V. Hoek, P. Somasundaran, F. Klaessig, V. Castranova and M. Thompson, Nat. Mater., 2009, 8, 543-557.
12 S. Tenzer, D. Docter, S. Rosfa, A. Wlodarski, J. Kuharev, A. Rekik, S. K. Knauer, C. Bantz, T. Nawroth, C. Bier, J. Sirirattanapan, W. Mann, L. Treuel, R. Zellner, M. Maskos, H. Schild and R. H. Stauber, ACS Nano, 2011, 5, 7155-7167.

13 C. D. Walkey, J. B. Olsen, H. Guo, A. Emili and W. C. W. Chan, J. Am. Chem. Soc., 2012, 134, 2139-2147.

14 P. Hinderliter, K. Minard, G. Orr, W. Chrisler, B. Thrall, J. Pounds and J. Teeguarden, Part. Fibre Toxicol., 2010, 7, $1-20$.

15 L. Rodriguez-Lorenzo, B. Rothen-Rutishauser, A. Petri-Fink and S. Balog, Part. Part. Syst. Charact., 2015, 32, 321-333.

16 S. Zhang, J. Li, G. Lykotrafitis, G. Bao and S. Suresh, Adv. Mater., 2009, 21, 419-424.

17 A. Ivask, I. Kurvet, K. Kasemets, I. Blinova, V. Aruoja, S. Suppi, H. Vija, A. Käkinen, T. Titma, M. Heinlaan, M. Visnapuu, D. Koller, V. Kisand and A. Kahru, PLoS One, 2014, 9, e102108.

18 C. J. Murphy and J. M. Buriak, Chem. Mater., 2015, 27, 4911-4913.

19 M. Mahmoudi, I. Lynch, M. R. Ejtehadi, M. P. Monopoli, F. B. Bombelli and S. Laurent, Chem. Rev., 2011, 111, 56105637.

20 M. Baalousha and J. R. Lead, Nat. Nanotechnol., 2013, 8, 308-309.

21 D. A. Urban, L. Rodriguez-Lorenzo, S. Balog, C. Kinnear, B. Rothen-Rutishauser and A. Petri-Fink, Colloids Surf., B, 2016, 137, 39-49.

22 D. E. Koppel, J. Chem. Phys., 1972, 57, 4814-4820.

23 B. J. Frisken, Appl. Opt., 2001, 40, 4087-4091.

24 A. Einstein, Ann. Phys., 1905, 17, 549-560.

25 A. Einstein, Ann. Phys., 1906, 324, 289-306.

26 A. Einstein, Ann. Phys., 1911, 339, 591-592.

27 P. Debye, Polar Molecules, Dover, New York, 1929.

28 R. Pecora, Dynamic Light Scattering: Applications of Photon Correlation Spectroscopy, Plenum Press, New York, 1985.

29 L. H. Hanus and H. J. Ploehn, Langmuir, 1999, 15, 3091-3100.

30 P. J. Patty and B. J. Frisken, Appl. Opt., 2006, 45, 2209-2216.

31 J. C. Thomas, J. Colloid Interface Sci., 1987, 117, 187-192.

32 S. Balog, L. Rodriguez-Lorenzo, C. A. Monnier, B. Michen, M. Obiols-Rabasa, L. Casal-Dujat, B. Rothen-Rutishauser, A. Petri-Fink and P. Schurtenberger, J. Phys. Chem. C, 2014, 118, 17968-17974.

33 D. Brogioli, D. Salerno, V. Cassina, S. Sacanna, A. P. Philipse, F. Croccolo and F. Mantegazza, Opt. Express, 2009, 17, 1222-1233.

34 A. Vailati, D. Asnaghi, M. Giglio and R. Piazza, Phys. Rev. E: Stat. Phys., Plasmas, Fluids, Relat. Interdiscip. Top., 1993, 48, R2358-R2361.

35 D. A. Giljohann, D. S. Seferos, W. L. Daniel, M. D. Massich, P. C. Patel and C. A. Mirkin, Angew. Chem., Int. Ed., 2010, 49, 3280-3294.

36 W. Zhou, X. Gao, D. Liu and X. Chen, Chem. Rev., 2015, 115, 10575-10636.

37 S. Balog, L. Rodriguez-Lorenzo, C. A. Monnier, M. ObiolsRabasa, B. Rothen-Rutishauser, P. Schurtenberger and A. S. Fink, Nanoscale, 2015, 7, 5991-5997. 
38 A. H. R. Koch, G. Lévêque, S. Harms, K. Jaskiewicz, M. Bernhardt, A. Henkel, C. Sönnichsen, K. Landfester and G. Fytas, Nano Lett., 2014, 14, 4138-4144.

39 H. G. Merkus, Particle Size Measurement: Fundamentals, Practice, Quality, Springer, 2009.

40 V. Filipe, A. Hawe and W. Jiskoot, Pharm. Res., 2010, 27, 796-810.

41 S. Jiang, K. Y. Win, S. Liu, C. P. Teng, Y. Zheng and M.-Y. Han, Nanoscale, 2013, 5, 3127-3148.

42 J. B. Haun, N. K. Devaraj, B. S. Marinelli, H. Lee and R. Weissleder, ACS Nano, 2011, 5, 3204-3213.

43 M. Lundqvist, J. Stigler, G. Elia, I. Lynch, T. Cedervall and K. A. Dawson, Proc. Natl. Acad. Sci. U. S. A., 2008, 105, 14265-14270.

44 J. Cohen, G. DeLoid, G. Pyrgiotakis and P. Demokritou, Nanotoxicology, 2013, 7, 417-431.

45 D. F. Moyano, K. Saha, G. Prakash, B. Yan, H. Kong, M. Yazdani and V. M. Rotello, ACS Nano, 2014, 8, 6748-6755.

46 S. Ritz, S. Schöttler, N. Kotman, G. Baier, A. Musyanovych, J. Kuharev, K. Landfester, H. Schild, O. Jahn, S. Tenzer and V. Mailänder, Biomacromolecules, 2015, 16, 1311-1321.

47 S. Stolnik, B. Daudali, A. Arien, J. Whetstone, C. R. Heald, M. C. Garnett, S. S. Davis and L. Illum, Biochim. Biophys. Acta, 2001, 1514, 261-279.

48 R. P. Carney, J. Y. Kim, H. Qian, R. Jin, H. Mehenni, F. Stellacci and O. M. Bakr, Nat. Commun., 2011, 2, 335.

49 V. Hirsch, C. Kinnear, L. Rodriguez-Lorenzo, C. A. Monnier, B. Rothen-Rutishauser, S. Balog and A. Petri-Fink, Nanoscale, 2014, 6, 7325-7331.
50 K. Larson-Smith and D. C. Pozzo, Langmuir, 2012, 28, 13157-13165.

51 S. Sekiguchi, K. Niikura, Y. Matsuo and K. Ijiro, Langmuir, 2012, 28, 5503-5507.

52 S. H. Brewer, W. R. Glomm, M. C. Johnson, M. K. Knag and S. Franzen, Langmuir, 2005, 21, 9303-9307.

53 D.-H. Tsai, F. W. DelRio, A. M. Keene, K. M. Tyner, R. I. MacCuspie, T. J. Cho, M. R. Zachariah and V. A. Hackley, Langmuir, 2011, 27, 2464-2477.

54 C. Passow, B. ten Hagen, H. Löwen and J. Wagner, J. Chem. Phys., 2015, 143, 044903.

55 M. Glidden and M. Muschol, J. Phys. Chem. C, 2012, 116, 8128-8137.

56 J. Rodríguez-Fernández, J. Pérez-Juste, L. M. Liz-Marzán and P. R. Lang, J. Phys. Chem. C, 2007, 111, 5020-5025.

57 M. Haghighi, M. A. Plum, G. Gantzounis, H.-J. Butt, W. Steffen and G. Fytas, J. Phys. Chem. C, 2013, 117, 84118419.

58 S. T. Moerz, A. Kraegeloh, M. Chanana and T. Kraus, ACS Nano, 2015, 9, 6696-6705.

59 K. C. Grabar, R. G. Freeman, M. B. Hommer and M. J. Natan, Anal. Chem., 1995, 67, 735-743.

60 K. R. Brown, D. G. Walter and M. J. Natan, Chem. Mater., 2000, 12, 306-313.

61 J. Turkevich, P. C. Stevenson and J. Hillier, Discuss. Faraday Soc., 1951, 11, 55-75.

62 B. Michen, C. Geers, D. Vanhecke, C. Endes, B. RothenRutishauser, S. Balog and A. Petri-Fink, Sci. Rep., 2015, 5, 9793. 